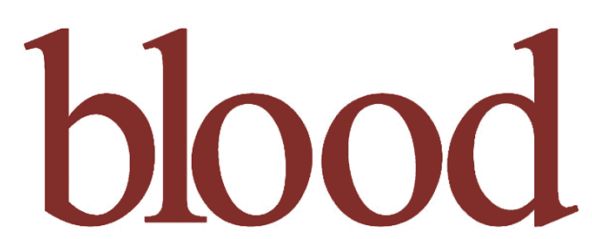

2009 113: 2375-2385

Prepublished online Dec 3, 2008;

doi:10.1182/blood-2007-09-113597

\title{
HOXA9 is required for survival in human MLL-rearranged acute leukemias
}

Joerg Faber, Andrei V. Krivtsov, Matthew C. Stubbs, Renee Wright, Tina N. Davis, Marry van den Heuvel-Eibrink, Christian M. Zwaan, Andrew L. Kung and Scott A. Armstrong

Updated information and services can be found at:

http://bloodjournal.hematologylibrary.org/cgi/content/full/113/11/2375

Articles on similar topics may be found in the following Blood collections:

Plenary Papers (255 articles)

Myeloid Neoplasia (93 articles)

Information about reproducing this article in parts or in its entirety may be found online at:

http://bloodjournal.hematologylibrary.org/misc/rights.dtl\#repub_requests

Information about ordering reprints may be found online at:

http://bloodjournal.hematologylibrary.org/misc/rights.dtl\#reprints

Information about subscriptions and ASH membership may be found online at:

http://bloodjournal.hematologylibrary.org/subscriptions/index.dtl

Blood (print ISSN 0006-4971, online ISSN 1528-0020), is published semimonthly by the American Society of Hematology, $1900 \mathrm{M} \mathrm{St}$, NW, Suite 200, Washington DC 20036.

Copyright 2007 by The American Society of Hematology; all rights reserved.

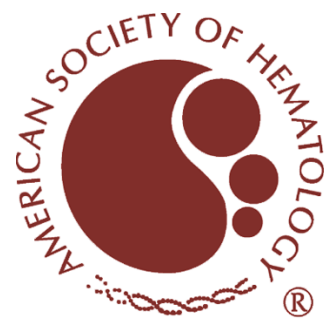




\title{
HOXA9 is required for survival in human $M L L$-rearranged acute leukemias
}

\author{
Joerg Faber, ${ }^{1}$ Andrei V. Krivtsov, ${ }^{1}$ Matthew C. Stubbs, ${ }^{1}$ Renee Wright, ${ }^{2}$ Tina N. Davis, ${ }^{2}$ Marry van den Heuvel-Eibrink, ${ }^{3}$ \\ Christian M. Zwaan, ${ }^{3}$ Andrew L. Kung, ${ }^{1,2}$ and Scott A. Armstrong 1,2,4 \\ 'Division of Hematology/Oncology, Children's Hospital, and 'Department of Pediatric Oncology and Harvard Medical School, Boston, MA; ${ }^{3}$ Division of Pediatric \\ Hematology/Oncology, Sophia Children's Hospital, Erasmus University Medical Center, Rotterdam, The Netherlands; and ${ }^{4}$ Harvard Stem Cell Institute, \\ Boston, MA
}

\begin{abstract}
Leukemias that harbor translocations involving the mixed lineage leukemia gene (MLL) possess unique biologic characteristics and often have an unfavorable prognosis. Gene expression analyses demonstrate a distinct profile for $M L L$-rearranged leukemias with consistent high-level expression of select Homeobox genes, including HOXA9. Here, we investigated the effects of HOXA9 suppression in MLLrearranged and $M L L$-germline leukemias using RNA interference. Gene expression
\end{abstract}

profiling after HOXA9 suppression demonstrated co-down-regulation of a program highly expressed in human MLLAML and murine MLL-leukemia stem cells, including HOXA10, MEIS1, PBX3, and MEF2C. We demonstrate that HOXA9 depletion in 17 human AML/ALL cell lines (7 MLL-rearranged, 10 MLL-germline) induces proliferation arrest and apoptosis specifically in MLL-rearranged cells $(P=.007)$. Similarly, assessment of primary AMLs demonstrated that HOXA9 suppression induces apoptosis to a greater extent in $M L L$-rearranged samples $(P=.01)$. Moreover, mice transplanted with HOXA9-depleted $\mathrm{t}(4 ; 11)$ SEMK2 cells revealed a significantly lower leukemia burden, thus identifying a role for HOXA9 in leukemia survival in vivo. Our data indicate an important role for HOXA9 in human $M L L$-rearranged leukemias and suggest that targeting HOXA9 or downstream programs may be a novel therapeutic option. (Blood. 2009;113:2375-2385)

\section{Introduction}

Translocations involving the mixed lineage leukemia locus ( $M L L$, All-1, HRX) on chromosome 11q23 are found in a variety of hematologic malignancies, including acute myeloid leukemias (AMLs), B-precursor and T-lineage acute lymphoblastic leukemias, and myelodysplastic syndrome. MLL rearrangements are present in most infant leukemias ${ }^{1-4}$ and in secondary leukemias after treatment with topoisomerase inhibitors. ${ }^{5-8}$ Infants diagnosed with lymphoblastic leukemia harboring a $M L L$ translocation respond poorly to current chemotherapy regimens and have a particularly unfavorable prognosis with an overall survival of less than $50 \%$. $^{9-13}$

The pathophysiologic mechanisms by which MLL translocations cause leukemia and the genes that serve as critical downstream targets during induction and maintenance of the leukemic phenotype are incompletely characterized. Gene expression profiling in human acute myeloid and lymphoblastic leukemias demonstrated a characteristic gene expression pattern for cases with $M L L$ rearrangements ${ }^{14-16}$ that may be driven by unique histone methylation programs. ${ }^{17-20} \mathrm{~A}$ common unifying feature in myeloid and lymphoid leukemias with $M L L$ rearrangements is high-level expression of Homeobox $(H O X)$ genes with a particular emphasis on the 5'-HOXA genes (HOXA5-11). ${ }^{14-16,21,22}$ Elevated expression of certain $5^{\prime}$-HoxA cluster genes is also found in murine leukemia models after introduction of various leukemia-associated Mll-fusion proteins. ${ }^{23-27}$ In a recent murine retroviral transduction/transplantation study, we determined the gene expression profile of leukemia stem cells that were initiated by expression of Mll-Af9 in committed

Submitted September 26, 2007; accepted October 23, 2008. Prepublished online as Blood First Edition paper, December 3, 2008; DOI 10.1182/blood2007-09113597.

An Inside Blood analysis of this article appears at the front of this issue. granulocyte macrophage progenitors (GMPs). ${ }^{28} 5^{\prime}$-HoxA cluster genes HoxA5, HoxA10, and in particular HoxA9 were prominent members of a gene expression signature found in leukemia stem cells and were immediately induced after Mll-Af9 expression. ${ }^{28}$ These findings support a hierarchical model of leukemia initiation by the $M L L-A F 9$ fusion where certain HOXA cluster genes belong to a crucial subset of proximate target genes, which are immediately activated by $M L L-A F 9$ expression. ${ }^{28,29}$ Further evidence that MLL fusion oncoproteins may directly regulate certain $H O X$ genes in hematopoietic cells emerges from recent studies in which wild-type MLL or MLL fusion proteins were found to be part of a multiprotein complex that regulates chromatin structure near the $5^{\prime}-H O X A$ cluster. $^{30-33}$

HOXA9 is directly involved in human leukemia caused by the translocation of HOXA9 with the gene encoding NUP98, a nucleoporin protein. ${ }^{34,35}$ Expression of this fusion protein immortalizes murine bone marrow cells and results in a myeloproliferative disorder and AML in bone marrow transplantation experiments. ${ }^{36,37}$ Retrovirally enforced overexpression of $H O X$ genes in murine and human marrow cells enhances expansion of hematopoietic cells in short-term in vitro cultures, and murine transplantation studies showed that forced expression of HoxA9 in hematopoietic precursors results in a significant expansion of hematopoietic stem cells and leukemia. ${ }^{38-43}$ Moreover, coexpressing HoxA9 suppresses Meis1-mediated apoptosis in a variety of murine and human leukemia cell types. ${ }^{44}$

During normal hematopoietic development, HOX genes are expressed in the stem cell and immature progenitor compartments
The online version of this article contains a data supplement.

The publication costs of this article were defrayed in part by page charge payment. Therefore, and solely to indicate this fact, this article is hereby marked "advertisement" in accordance with 18 USC section 1734.

(C) 2009 by The American Society of Hematology 
of bone marrow but are transcriptionally down-regulated on induction of terminal differentiation. ${ }^{45-47}$ Homozygous HoxA9deficient mice are viable but exhibit a variety of mild hematopoietic defects, including reduced number of committed myeloid and B-cell progenitors and a blunted neutrophilic response to granulocyte colony-stimulating factor. ${ }^{48,49}$ HoxA9-deficient hematopoietic stem cells (HSCs) demonstrate an impaired proliferative response to cytokines in vitro and defective competitive repopulating activity in vivo. ${ }^{50}$ Thus, HoxA9 does appear to play a role in normal HSC biology, but is not essential for HSC development.

Although most studies point to some role for $H O X$ genes in myeloid leukemogenesis, it remains unclear whether continued expression of any $H O X A$ cluster gene is necessary for survival of AMLs. Murine studies addressing this issue have provided mixed results. $M l l-E n l$-transduced murine myeloid progenitors deficient for HoxA9 demonstrate significantly compromised self-renewal potential in vitro and impaired development of leukemias when transplanted in vivo. ${ }^{27}$ However, HoxA9-1deficient progenitors transduced with $M l l-G a s 7$ are capable of initiating leukemia, ${ }^{51}$ and $M l l-A f 9^{+}, H o x A 9^{-1-}$ mice develop leukemia with a similar frequency and latency as mice carrying Mll-Af9 with germline HoxA9. ${ }^{24}$ These studies raise the issue as to whether there is compensation for the lack of HoxA9 during development, and prompt assessment of an acute loss of HOXA9 expression in a fully developed leukemia. In this study, we aimed to further elucidate the role of HOXA9 expression in human $M L L$-rearranged and nonrearranged leukemias. Through use of a small hairpin RNA (shRNA)-mediated RNA interference approach and subsequent gene expression profiling analysis, we demonstrate a marked impact of HOXA9 suppression on survival of human $M L L$-rearranged leukemia cells associated with tight co-down-regulation of genes that were part of a MLL signature in primary human samples.

\section{Methods}

\section{Generation of murine leukemia stem cells}

Murine leukemic granulocyte macrophage progenitors (L-GMPs) were generated as described previously ${ }^{28}$ (Document S1, available on the Blood website; see the Supplemental Materials link at the top of the online article). For HOXA9 shRNA knockdown experiments, IL-7R ${ }^{-} \mathrm{Lin}^{-} \mathrm{Sca}-1^{-} \mathrm{c}-$ $\mathrm{Kit}^{+} \mathrm{CD} 34^{+}$-FcgRII/III ${ }^{+}$GMP-like leukemic cells (L-GMPs) sorted from leukemic mice were incubated with lentiviral shRNA vectors and plated in methylcellulose media M3234 or liquid culture (Myelocult M5300; StemCell Technologies, Vancouver, BC) supplemented with $10 \mathrm{ng} / \mathrm{mL}$ IL-3 (PeproTech, Rocky Hill, NJ), 1× penicillin/streptomycin (Invitrogen, Carlsbad, CA). All mouse studies were approved by the Dana Farber Cancer Institute Institutional Animal Care and Use Committee.

\section{Leukemia primary patient samples}

Pediatric AML samples were obtained from the Erasmus MC, Sophia Children's Hospital (Rotterdam, The Netherlands). Approval was obtained from the Erasmus MC Institutional Review Board for these studies. Informed consent was obtained in accordance with the Declaration of Helsinki. Analyzed samples consisted of cryopreserved cells of AML patients with confirmed rearrangement of the $M L L$ locus determined by either cytogenetic analysis, fluorescence in situ hybridization, or Southern blot and AML patients with $M L L$ germline karyotype. Patient samples 1, 2, 3, 5, and 6 harbored a $\mathrm{t}(9 ; 11)$. Sample 4 possessed a $\mathrm{t}(11 ; 19)$. Mean percentage of blasts was approximately $90 \%$. Although a variable number of dead cells was observed after thawing in the same range as previously demonstrated, ${ }^{52}$ culture conditions were optimized to support $50 \%$ to $60 \%$ viable cells at 72 to 96 hours for subsequent phenotypic assays (see Document S1).

\section{Transfection with shRNA constructs}

shRNA cloned into hairpin pLKO.1-lentiviral vector were kindly synthesized by the Harvard/MIT Broad Institute RNA consortium using a complex selection algorithm to minimize off-target effects (http://www.broad.mit.edu/ rnai/trc). Lentiviral supernatants were obtained in $293 \mathrm{~T}$ cells by cotransfection of the shRNA plasmids, packaging plasmids containing gag, tat, and rev genes, and a plasmid expressing VSV-G. For lentiviral infection of leukemia cell lines or primary AML patient cells, $10^{5}$ cells were seeded in 96-well tissue culture plates (100 $\mu \mathrm{L}$ per well) in the appropriate culture media. Polybrene (hexadimethrine bromide; Sigma-Aldrich, St Louis, MO) was added at a final concentration of $7 \mu \mathrm{g} / \mathrm{mL}$. After adding $150 \mu \mathrm{L}$ lentiviral particles (titer of each lentiviral shRNA construct adjusted to $1.6 \times 10^{6}$ transducing units $/ \mathrm{mL}$ to achieve a multiplicity of infection of 2.4 transducing units/cell), spinoculation was performed at $2250 \mathrm{~g}$ for 90 minutes at $37^{\circ} \mathrm{C}$. Eight hours after infection, the media was replaced with $100 \mu \mathrm{L}$ of the appropriate fresh culture media. If puromycin selection was desired, puromycin dihydrochloride (Sigma-Aldrich) at a final concentration of $2.5 \mu \mathrm{g} / \mathrm{mL}$ was added. Cells were incubated at $37^{\circ} \mathrm{C}, 5 \% \mathrm{CO}_{2}$ until use for downstream applications (see Document S1).

\section{Analysis of cell viability and apoptosis}

To assay cell viability, we used the colormetric diphenyltetrazolium bromide (MTT) Cell Proliferation Kit I (Roche Diagnostics, Indianapolis, IN) according to the instructions of the manufacturer and flow cytometric analysis after fluorescein isothiocyanate-annexin V (BioVision, Mountain View, CA)/propidium iodide (Invitrogen) double staining. Cell-cycle analysis was performed by flow cytometric analysis of propidium iodidestained cells at defined time points after shRNA transduction.

\section{Murine transplantation experiments and in vivo imaging}

Luminescent $\mathrm{t}(4 ; 11)$ SEMK2-M1 cells were generated, and bioluminescent in vivo imaging was performed as previously described ${ }^{53}$ (Document S1). For further postmortem analysis of mouse leukemia burden, single-cell suspensions were generated from spleen. Red blood cells were lysed on ice using red blood cell lysis solution (Gentra Systems, Minneapolis, MN) for 5 minutes. After centrifugation, cells were resuspended in phosphatebuffered saline supplemented with $10 \%$ fetal bovine serum. A total of $5 \times 10^{8}$ of nucleated cells were incubated 40 minutes on ice with $10 \mu \mathrm{L}$ of each of the following fluorochrome-conjugated antibodies: mouse antihuman CD19-TC (Invitrogen), anti-mouse CD45.2-fluorescein isothiocyanate (BD Biosciences, San Jose, CA). After a double wash in phosphatebuffered saline, the cell suspension was sorted using FACSAria multicolor cell sorter (BD Biosciences).

\section{Data analysis and statistical methods}

After hybridization to Affymetrix Human Genome U133A2.0 microarrays (Santa Clara, CA), data were analyzed using the GenePattern 3.0 software package ${ }^{54}$ including the Gene set enrichment analysis (GSEA) module ${ }^{55}$ (Broad Institute; Document S1). All other statistical calculations were performed using SPSS 14.0 software package (SPSS, Chicago, IL). The original microarray data have been deposited into the Gene Expression Omnibus database, under accession number GSE13714.

\section{Results}

\section{Establishment and validation of HOXA9 suppression in human leukemia cells}

To establish efficient knockdown targeting the human HOXA9 mRNA, 5 different shRNA constructs (1F2-HOXA9shRNA, 1F3HOXA9shRNA, 2A4-HOXA9 shRNA, 2A5-HOXA9shRNA, and 
Figure 1. ShRNA-mediated knockdown of HOXA9 expression in human $t(9 ; 11)$ MOLM-14 and $t(4 ; 11)$ SEMK2 cells and murine leukemia stem cells (L-GMP). (A) Quantitative real-time PCR analysis of HOXA9 expression 72 hours after transduction of MOLM-14 and SEMK2 cells with 1 of 4 different HOXA9 shRNA constructs (1F2-HOXA9shRNA, 1F3-HOXA9shRNA, 2A4HOXA9shRNA, and 2A5-HOXA9shRNA) without puromycin selection. A highly efficient HOXA9 suppression is demonstrated compared with a nontargeting control shRNA directed toward GFP. Western blot analysis 72 hours after transduction confirmed HOXA9 protein knockdown. (B) HoxA9 depletion in highly purified murine leukemia stem cells (L-GMP) resulted in a progressive induction of apoptosis in liquid culture compared with cells transduced with a control shRNA. (C) Analysis of L-GMP colony formation and replating capacity after HoxA9 suppression. Colonies were counted and replated at day 8 . A significant decrease in colony number and replating capacity is demonstrated for L-GMPs transduced with HoxA9-directed shRNA without puromycin selection. (D) Analysis of L-GMP colony formation after HOXA9 suppression in a mixed-populations assay. L-GMPs were transduced with 1F2-HOXA9shRNA, 1F3-HOXA9shRNA, or GFPcontrol shRNA constructs, and populations of HOXA9shRNA or GFP-control shRNA-transduced cells were mixed in a ratio of 9:1 (HOXA9shRNA-transduced cells/GFP-control shRNA-transduced cells) and plated in semisolid medium. The total number of colonies at day 8 is shown for the mixed populations (1F2HOXA9shRNA/GFP-control-shRNA; 1F3-HOXA9shRNA/GFPcontrol-shRNA) and the controls containing only GFP-control shRNA-transduced cells. (E) Quantitative real-time PCR further demonstrated similar HOXA9 expression in colonies that arose from the mixed HOXA9shRNA/GFP-control shRNA populations and controls containing only GFP-control shRNA-transduced cells (day 8 ).
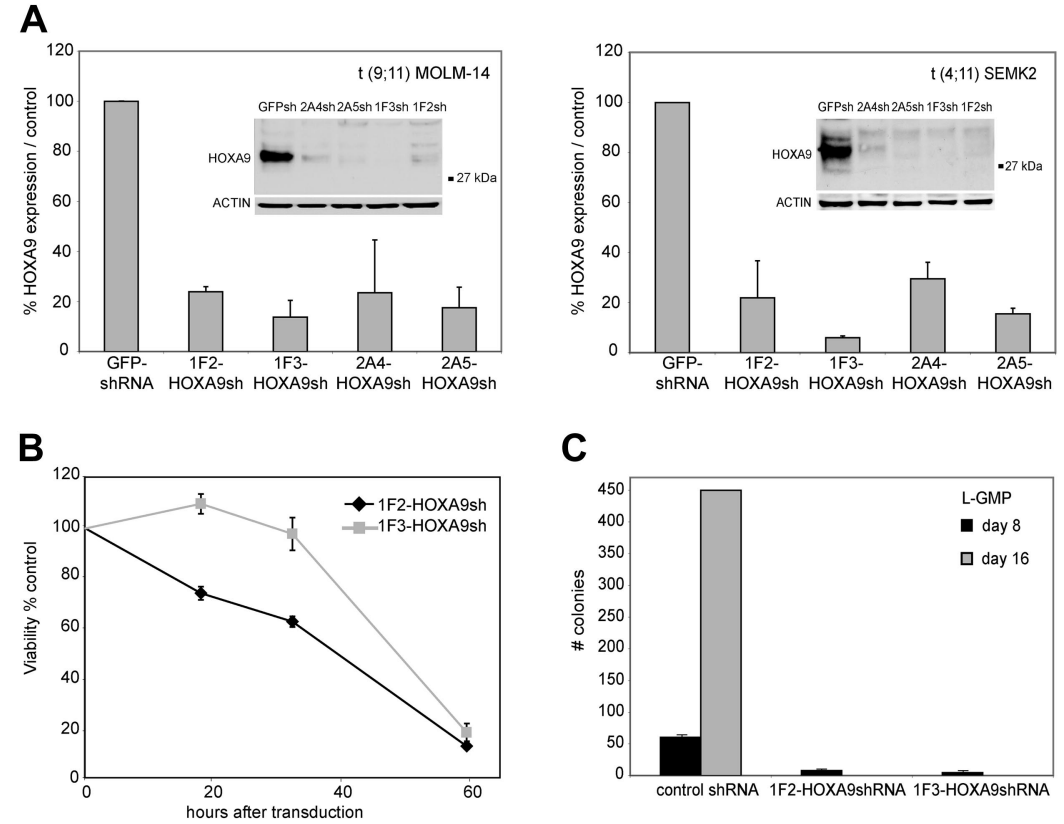

\section{C}

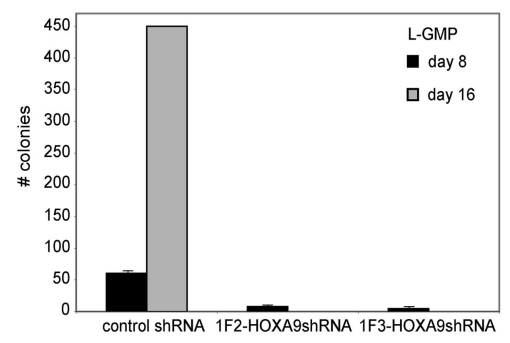

E

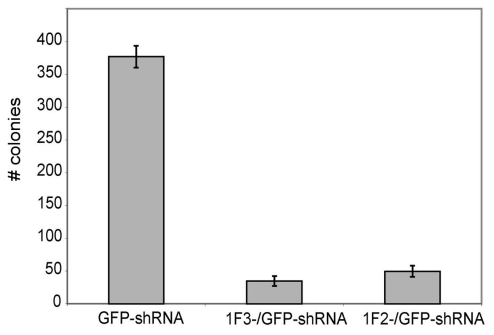

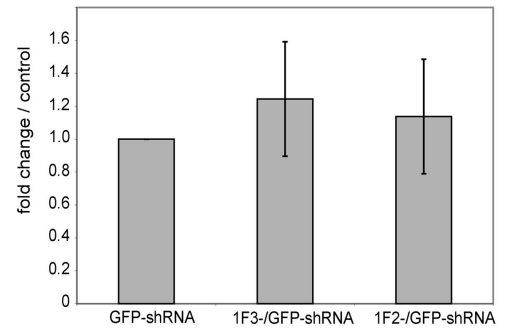

2A6-HOXA9shRNA) were stably introduced into target cells of interest using a lentiviral vector system. The shRNA sequences were selected using an algorithm that enhances the likelihood of efficient RNA interference (RNAi) and minimizes targeting of other mRNAs. ${ }^{56}$ Genome-wide blast search demonstrated at least 6 sequence mismatches for any other gene in the human genome database. First, we assessed transduction efficiency using the green fluorescent protein (GFP)-control shRNA construct in 17 different $M L L$-rearranged and $M L L$ wild-type leukemia cell lines by selection in puromycin and demonstrated a high percentage of puromycin resistance ( $>80 \%$ transduction efficiency), which was similar in each cell line tested (Figure S1), whereas nontransduced control cells of each type were effectively eliminated (not shown). Thus, all subsequent experiments were performed without puromycin selection. We then introduced each of the HOXA9 targeting shRNAs into $\mathrm{t}(9 ; 11)$ MOLM-14 cells and t(4;11) SEMK2-M1 (SEMK2) cells and compared the suppression efficiency to cells that were transduced with either a nontargeting control shRNA directed toward GFP, an empty lentiviral vector, or untreated. Using quantitative real-time polymerase chain reaction (PCR), we found that 3 of 5 HOXA9shRNA constructs (1F2-HOXA9shRNA, 1F3HOXA9shRNA, and 2A5-HOXA9shRNA) suppressed HOXA9 RNA levels by at least $80 \%$ compared with control. The 2A4HOXA9shRNA construct showed a more variable effect $(60 \%$ $80 \%$ suppression; Figure 1A), and the 2A6-HOXA9shRNA had only minimal effect (not shown) and was therefore excluded from further experiments. The efficacy of HOXA9 suppression by the 4 effective constructs was confirmed by Western blot analysis (Figure 1A). Because introduction of some RNAi constructs has been previously found to unspecifically induce interferon response, ${ }^{57}$ we next analyzed expression changes in the interferon response genes $O A S 1, I F I T M 1$, and $M X 1$ by quantitative real-time PCR as suggested in recent guidelines. ${ }^{58}$ Transduction of any of the lentiviral constructs showed no induction of the interferon response genes analyzed with expression levels similar to untreated control cells (Figure S2). Thus, we have validated 4 independent shRNA constructs that suppress HOXA9 expression in MLLAF9 and MLLAF4 human leukemia cell lines.

\section{Effects of HoxA9 knockdown in murine leukemia stem cells}

In our recent murine $M l l-A f 9$ transduction/transplantation study, gene expression profiling revealed aberrant expression of HoxA9 as part of a stem cell signature in a $\mathrm{IL}_{-7 \mathrm{R}^{-} \mathrm{Lin}^{-} \mathrm{Sca}-1^{-} \mathrm{c}-}$ $\mathrm{Kit}^{+} \mathrm{CD} 34^{+} \mathrm{FcgRII} / \mathrm{III}{ }^{+}$GMP-like (L-GMP) leukemia stem cell population. ${ }^{28}$ We therefore first explored the effects of HoxA9 depletion in this well-defined and previously characterized murine leukemia stem cell population. To achieve maximum HoxA9 knockdown in murine L-GMP, the 2 most effective HOXA9 shRNA constructs with $100 \%$ target sequence homology between the human and murine HOXA9 gene were selected (1F2-HOXA9shRNA, 1F3-HOXA9shRNA). These constructs had already been previously successfully used in murine hematopoietic cells. ${ }^{59}$ Using quantitative real-time PCR, we found that both shRNA constructs efficiently suppressed HOXA9 RNA levels in L-GMP compared with cells transduced with a nontargeting control shRNA (Figure S3). L-GMP cells were 
A

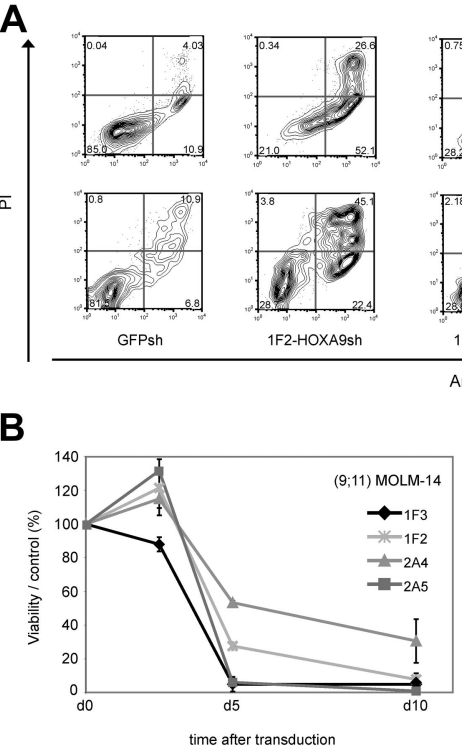

C

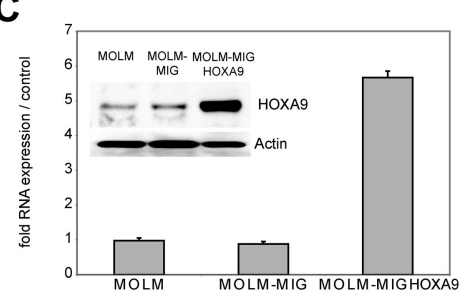

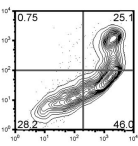
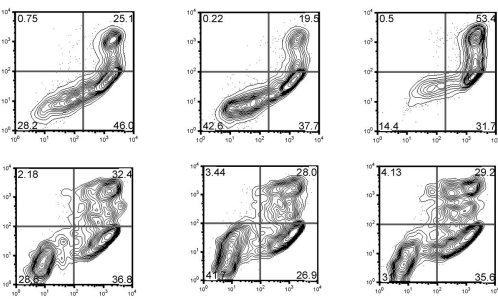

Annexin V

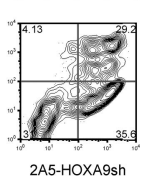

$t(9 ; 11)$ MOLM-1

4 ;11) SEMK2

Figure 2. Analysis of phenotypic consequences after shRNAmediated HOXA9 suppression in $\mathrm{t}(9 ; 11)$ MOLM-14 AML and $\mathbf{t}(4 ; 11)$ SEMK2 ALL cells. (A) Analysis of cell viability as measured by annexin V/PI positivity at day 7 after transduction of $\mathrm{t}(9,11)$ MOLM-14 cells and $\mathrm{t}(4 ; 11)$ SEMK2 cells with 1 of 4 HOXA9 targeted shRNAs or GFP control shRNA. The percentage of viable (annexin V/PI negative) cells is shown in the HOXA9shRNA and GFP control shRNA-transduced group. (B) Time course analysis of cell viability as measured by annexin V/PI positivity after HOXA9 suppression in an independent experiment. Cells were transduced with either 1 of 4 HOXA9 targeted shRNAs or GFP-control shRNA. The data are graphed as percentage of viable cells that were transduced with HOXA9shRNA/percentage of viable cells that were transduced with GFP-control shRNA at a given time point. These results were confirmed 3 times. (C) Quantitative real-time PCR and Western blot analysis of ectopic HOXA9 overexpression in $\mathrm{t}(9 ; 11)$ MOLM-14 cells compared with untransduced cells and cells transduced with the empty vector construct. (D) MOLM-14 cells ectopically overexpressing HOXA9 were transduced with the 2A4-HOXA9shRNA construct specifically targeting the 3 '-untranslated region of endogenous HOXA9 or the GFP-control shRNA construct. Ectopic overexpression of HOXA9 almost completely rescued the phenotype (annexin V/PI staining).

transduced with either the 1F2-HOXA9shRNA or the 1F3HOXA9shRNA construct, and viability in liquid culture supplemented with cytokines was compared with L-GMP cells that were transduced with a nontargeting control shRNA. Whereas control shRNA-transduced cells survived in liquid culture, L-GMPs transduced with either the 1F2-HOXA9shRNA or 1F3-HOXA9shRNA construct showed a progressive induction of cell death with onset short after transduction, leading to almost complete loss of viable cells at day 3 (Figure 1B). Next, we analyzed colony-forming and replating capacity of L-GMPs transduced with 1F2-HOXA9shRNA, 1F3-HOXA9shRNA, or control shRNA constructs in semisolid medium. At day 8, colony formation was significantly reduced in the 2 L-GMP populations transduced with either the 1F2-HOXA9shRNA or 1F3-HOXA9shRNA construct demonstrating a colony count of less than $10 \%$ of the control shRNA treated group (Figure 1C). Moreover, replating and reevaluation at day 16 revealed a more than 10-fold expansion in the control L-GMP population, whereas colony-forming activity was completely abrogated in the HoxA9 shRNA-transduced populations (Figure 1C). Furthermore, analysis of colony-forming capacity of L-GMP in a mixed-populations assay demonstrated similar findings: LGMPs were transduced with the 1F2-HOXA9shRNA, 1F3HOXA9shRNA, or GFP-control shRNA construct. Cells were then mixed in a ratio of 9:1 (HOXA9shRNA-transduced cells: GFP-control shRNA-transduced cells) and plated in semisolid medium with cytokines. At day 8 , a significantly lower number of colonies was observed in the 1F2-HOXA9shRNA/GFPcontrol shRNA and 1F3-HOXA9shRNA/GFP-control shRNA mixed populations compared with controls containing only cells transduced with GFP-control-shRNA (Figure 1D). Quantitative real-time PCR further demonstrated HOXA9 expression levels in the mixed populations similar to those of the controls (Figure 1E), indicating that only cells with nonsuppressed HOXA9 expression were capable of colony formation. These findings point toward a central role for HoxA9 in the maintenance of murine $M l l$-rearranged leukemia stem cells. We therefore sought to further characterize the effects of HOXA9 depletion in human leukemia cell lines and primary leukemia patient samples.

\section{Effects of HOXA9 suppression in human MLLAF4 SEMK2 and MLLAF9 MOLM-14 leukemia cells}

To examine the effect of HOXA9 suppression on the cellular phenotype in human leukemia cells, we first analyzed apoptosis induction as measured by annexin V/PI positivity after transduction of $\mathrm{t}(9,11)$ MOLM-14 AML M5 and $\mathrm{t}(4 ; 11)$ SEMK2 ALL cells with each of the 4 HOXA9 targeted shRNAs or GFPcontrol shRNA. HOXA9 suppression led to a significant reduction of cell viability with all 4 HOXA9shRNA constructs compared with control (Figure 2A). In an independent experiment, time course analysis of apoptosis induction further demonstrated progressive reduction of cell viability starting 72 to 84 hours after transduction (Figure 2B). Induction of cell death was preceded by inhibition of proliferative capacity as assessed by MTT assay approximately 24 hours before induction of cell death became evident (48 hours after transduction; Figure S4).

Next, to further assess specificity of the observed correlation between HOXA9 suppression and induction of apoptosis, GFPHOXA9 was ectopically overexpressed in MOLM-14 cells. GFP-positive cells were sorted and HOXA9 overexpression was confirmed by quantitative real-time PCR and Western blot analysis (Figure 2C). Transduction with the 2A4-HOXA9shRNA 
From www.bloodjournal.org at Erasmus MC Medical Library on June 16, 2009. For personal use only.

A

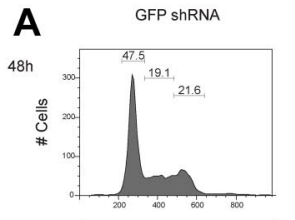

$72 \mathrm{~h}$

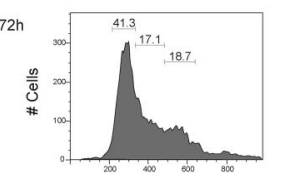

$96 \mathrm{~h}$

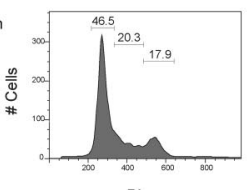

1F3-HOXA9 shRNA
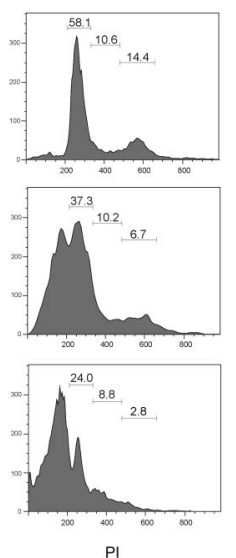

B
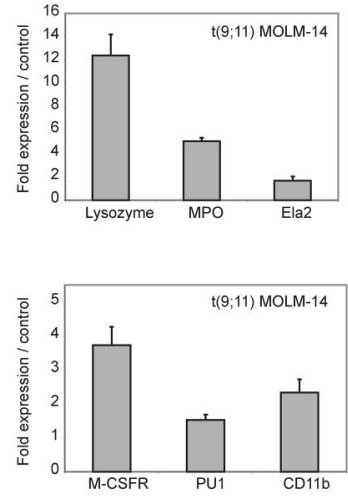

E

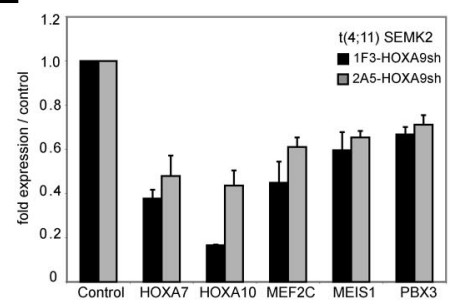

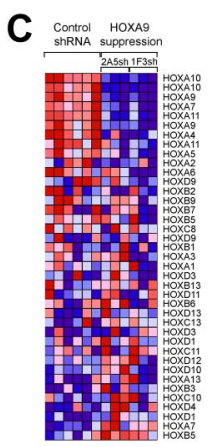

D MLL-rearranged AML

MLL-wildtype AML

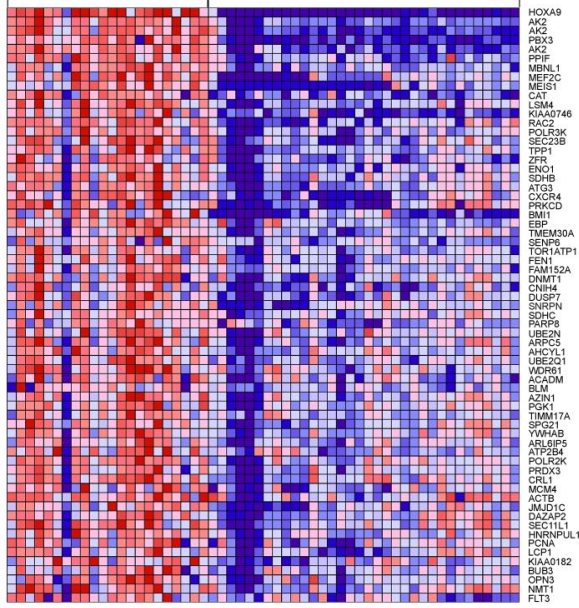

Figure 3. Effects of HOXA9 suppression in human $t(9 ; 11)$ MOLM-14 and $t(4 ; 11)$ SEMK2 cells. (A) Fluorescence-activated cell sorter analysis of cell-cycle status after transduction of MOLM-14 cells with 1F3-HOXA9shRNA after PI staining. A progressive increase of cells in $\mathrm{G}_{1}$ phase and a decrease of cells in $\mathrm{G}_{2}$ and $\mathrm{S}$ phases were noted compared with control GFP-shRNA-transduced cells with onset 24 to 36 hours before induction of apoptosis. (B) Analysis of mRNA expression of myeloid differentiation markers M-CSF1R, PU.1, and ITGAM (CD11b) and the granule molecules lysozyme (LYZ), myeloperoxidase (MPO), and elastaseA2 (ELA2) associated with terminal myeloid differentiation by quantitative real-time PCR 72 hours after HOXA9shRNA transduction. Compared with control GFP-shRNA transduced cells, a significant induction of mRNA expression was observed. (C) Gene expression analysis 44 hours after HOXA9 suppression in t(9;11) MOLM-14 cells with 2 different HOXA9shRNAs (1F3-HOXA9shRNA; 2A5-HOXA9shRNA) in triplicates compared with GFP-shRNA-transduced controls. Analysis of concomitant expression changes in other Homeobox $A$ - $D$ cluster genes demonstrated co-down-regulation of 5'-HOXA cluster gens in the HOXA9 suppression signature. (D) GSEA of the top 500 genes down-regulated after HOXA9 suppression in a gene expression dataset of 56 human AML patients. ${ }^{60}$ The HOXA9 suppression gene set is significantly enriched in a signature previously identified as more highly expressed in MLL-AML compared with other genetically defined AML subtypes $(E S=0.48 ; P<.001)$. (E) Confirmation of co-down-regulation of $5^{\prime}$-HOXA cluster genes as well as the HOXA9 cofactors MEIS1 and PBX3 and the transcription factor MEF2C in t(4;11) SEMK2 cells (quantitative real-time PCR analysis).

construct to specifically target the $3^{\prime}$-untranslated region of endogenous HOXA9 demonstrated almost complete rescue of the phenotype with a high level of protection by ectropic overexpression of nontargetable HOXA9 compared with controls (Figure 2D). Rescue of the phenotype by ectopically expressing a nontargetable HOXA9 construct was also confirmed in an independent experiment in $\mathrm{t}(9 ; 11)$ THP-1 cells applying siRNA-mediated HOXA9 suppression (Figure S5).

To further assess the effects on proliferation and viability after HOXA9 depletion, we analyzed cell cycle status. A time course analysis of MOLM-14 cells transduced with the 1F3HOXA9shRNA construct revealed a progressive increase of cells in the $G_{1}$ phase and a decrease in $G_{2}$ and $S$ phases, 24 to 36 hours before the prominent induction of cell death (Figure $3 \mathrm{~A})$. These findings are suggestive of an accumulation of the HOXA9shRNA-transduced cell population in $\mathrm{G}_{1}$ phase before progressively undergoing apoptosis. We next analyzed whether HOXA9 depletion might induce differentiation before apoptosis.
Analysis of mRNA expression of myeloid differentiation markers M-CSF1R, PU.1, and ITGAM (CD11b) and granule molecules associated with terminal myeloid differentiation (lysozyme [LYZ]), myeloperoxidase [MPO]), elastaseA2 [ELA2]) by quantitative real-time PCR demonstrated a significant induction of these markers 72 hours after HOXA9shRNA transduction compared with GFP shRNA-transduced control cells (Figure 3B). Fluorescence-activated cell sorter analysis of the myeloid differentiation marker CD14 in MOLM-14 cells revealed a significant increase in CD14 expression in cells transduced with HOXA9 shRNA constructs compared with controls (Figure S6). Induction of CD14 expression was detectable as early as 48 hours after shRNA transduction and reached a peak at 84 hours (GFP shRNA, 1.62\%; 1F3-HOXA9shRNA, $14.61 \%$; 2A5-HOXA9shRNA, 17.58\%) shortly before progressive induction of cell death set in (Figure S6). These data indicate that HOXA9 depletion initiates some degree of cellular differentiation before induction of apoptosis. 
A
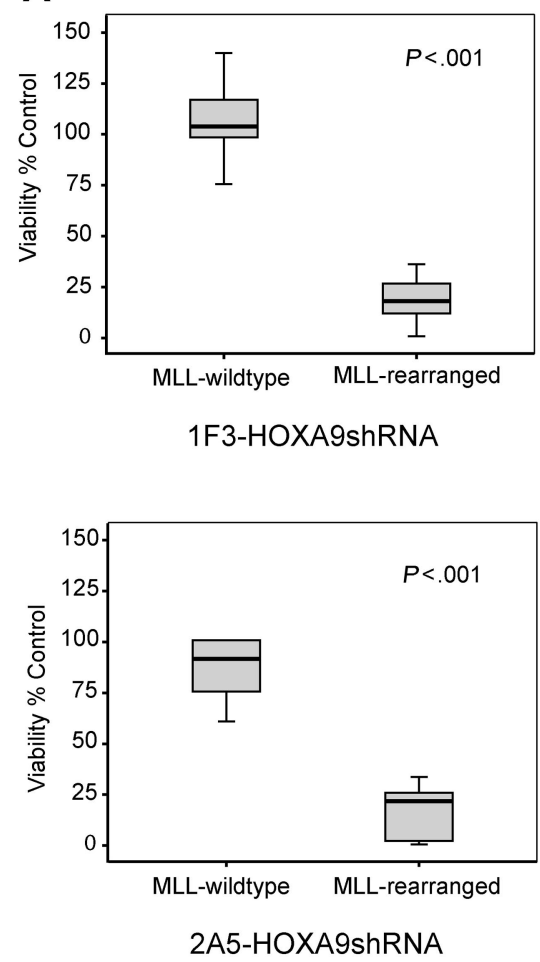
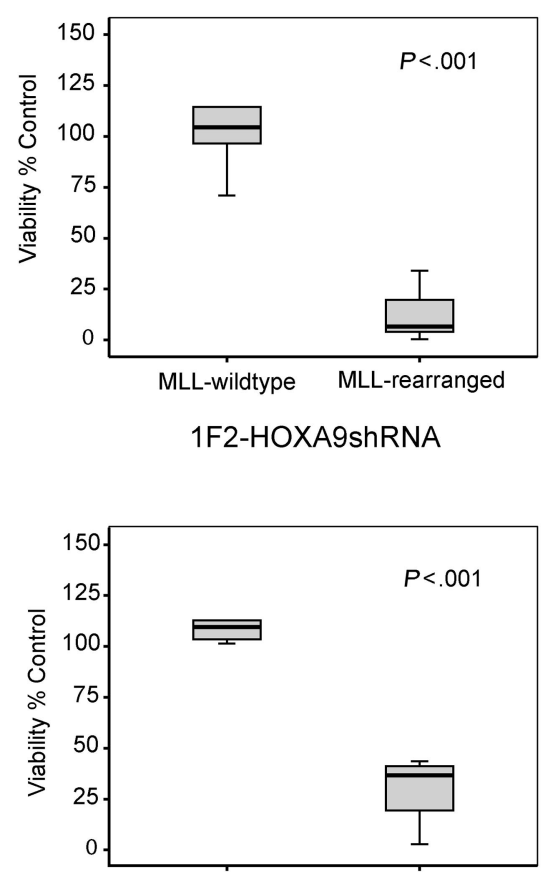

MLL-wildtype MLL-rearranged
B

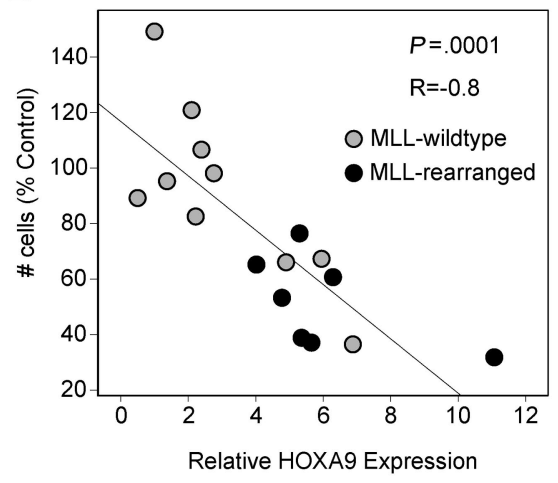

Figure 4. Induction of apoptosis by HOXA9 suppression is significantly correlated with the presence of the $M L L$-fusion oncogene. (A) Analysis of cell viability at day 7 after HOXA9 shRNA transduction in a larger panel of $11 \mathrm{AML} / \mathrm{ALL}$ cell lines (6 MLL-rearranged; 5 MLL-wild-type) showing significantly higher apoptosis induction in MLL-rearranged cells with all 4 HOXA9shRNA constructs. (B) Independent analysis in a panel of 17 AML/ALL cell lines (7 MLL-rearranged; $10 \mathrm{MLL}$-wild-type) further demonstrated a significant correlation between cell number and baseline HOXA9 expression after shRNA-mediated suppression, with the greatest effect in cell lines expressing the highest HOXA9 levels $(\mathrm{R}=-0.8: P=.001 ; \bullet, M L L$-rearranged cell lines; $\bigcirc, M L L$-wild-type cell lines).

\section{Gene expression analysis after HOXA9 suppression}

Next, we used gene expression profiling to characterize changes that occur after HOXA9 suppression in $\mathrm{t}(9 ; 11)$ MOLM-14 cells. RNA was purified 44 hours after transduction in triplicates with 2 of the 2 most effective HOXA9shRNA constructs $(3 \times 1 \mathrm{~F} 3$ HOXA9shRNA; $3 \times 2$ A5-HOXA9shRNA) or GFP-control shRNA $(6 \times)$ and hybridized to Affymetrix Human Genome U133A2.0 microarrays. Initial comparative marker analysis of expression changes applying either one of the HOXA9shRNAs against GFP-control shRNA identified large groups of genes with decreased expression in cells treated with both HOXA9directed shRNA constructs. GSEA ${ }^{55}$ further demonstrated almost complete overlap of the top 500 gene probes with decreased expression after HOXA9 suppression applying the 2 different HOXA9-shRNAs (enrichment score [ES] $=0.82$, $P<.001$; Figure S7A). Having established that both HOXA9shRNAs led to almost identical expression changes after HOXA9 suppression, both datasets were combined for further analysis. Among the genes with the most significantly decreased expression after HOXA9 suppression were genes previously identified as important in leukemogenesis and self-renewal of leukemia stem cells, such as the HOXA9 cofactor MEIS1, $M E F 2 C$, a member of the myocyte enhancer factor 2 gene family of transcription factors and BMII (Figure S7B). We then aimed to determine whether HOXA9 suppression leads to concomitant changes in other Homeobox $A-D$ cluster genes. Assessment of expression changes in a panel of Homeobox $A-D$ cluster genes demonstrated concomitant co-down-regulation of a subset of $5^{\prime}-H O X A$ cluster genes, including HOXA7 and
HOXA10 (Figure 3C). Next, to assess the relevance of the HOXA9 suppression signature in primary human AML, we determined whether there was significant overlap between our data and a gene expression signature in primary human $M L L$ rearranged AML. ${ }^{60}$ GSEA demonstrated significant overlap between the top 500 genes that decreased in expression after HOXA9 suppression (Figure S7B) and genes more highly expressed in human MLL-AML compared with other genetically defined AML subtypes ( $\mathrm{ES}=0.48 ; P<.001$; Figure $3 \mathrm{D})$. These overlapping gene sets further validated genes previously implicated in the pathogenesis of $M L L$-rearranged leukemias, including the HOXA9 cofactors MEIS1 and PBX3,MEF2C, and $B M I 1$ (Figure 3D). Co-down-regulation of 5'-HOXA-cluster genes and the HOXA9 cofactors MEIS1 and $P B X 3$ as well as the myocyte enhancer factor 2c $(M E F 2 C)$ after HOXA9 suppression was also demonstrated in $\mathrm{t}(4 ; 11)$ SEMK2 cells (Figure $3 \mathrm{E}$ ). These data demonstrate an important role for HOXA9 as an upstream regulator of an $M L L-A F 9$-associated gene expression program.

\section{Effect of HOXA9 suppression in human leukemia cell lines and primary human leukemias}

Next, we analyzed whether the observed induction of apoptosis after HOXA9 suppression is specific for $\mathrm{t}(9 ; 11)$ MOLM-14 and $\mathrm{t}(4 ; 11)$ SEMK2 cells or whether a similar phenotype can also be identified in an extended panel of $M L L$-rearranged and $M L L$ wild-type cell lines. In a panel of 11 AML/ALL cell lines (6 $M L L$-rearranged; $5 M L L$-wild-type), a significantly higher induction of cell death was demonstrated in the $M L L$-rearranged 
Figure 5. HOXA9 suppression induces apoptosis in primary $M L L$-rearranged human AML cells. (A) Analysis of baseline HOXA9 expression before shRNA transduction by quantitative real-time PCR. (B) The mean HOXA9 expression level was found to be significantly higher in primary AML patient samples bearing an $M L L$ translocation $(P=.004)$. (C) Analysis of apoptosis induction by annexin $\mathrm{V}$ staining 72 hours after transduction with the 1F3-HOXA9shRNA or control shRNA. (D) Induction of apoptosis is significantly higher in the MLLrearranged group $(P=.010)$. (E) A significant correlation between baseline HOXA9 expression and impact of HOXA9 knockdown on viability is demonstrated. Samples expressing high-level HOXA9 were found to be especially susceptible to HOXA9 depletion $(\mathrm{R}=-0.8$; $P=.005)$.
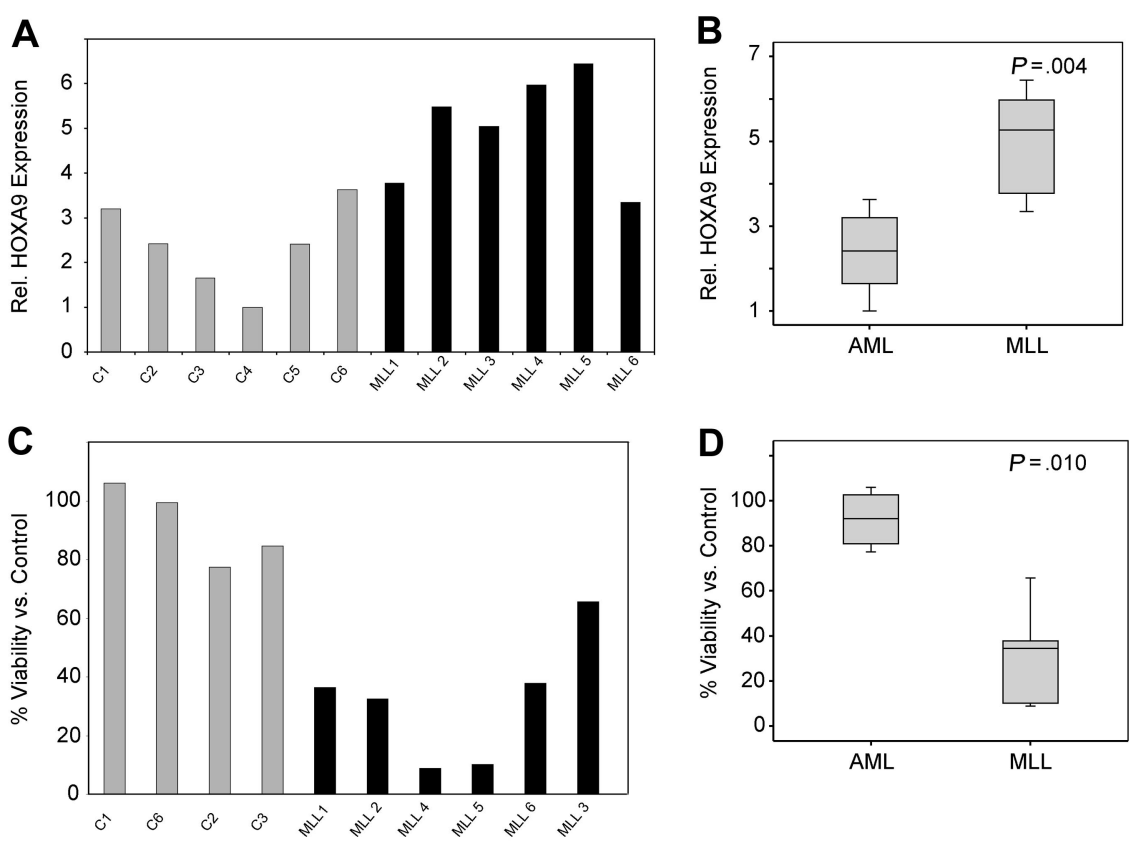

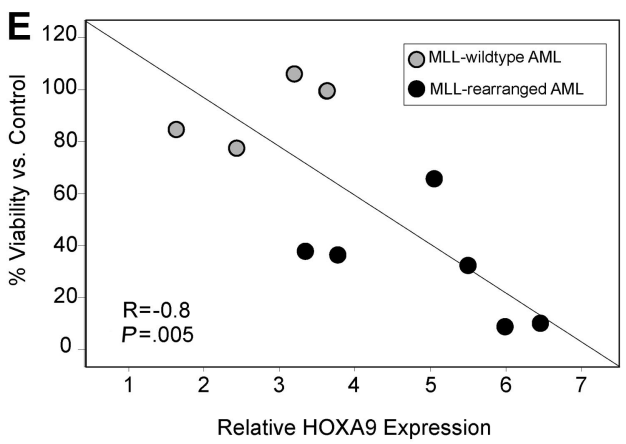

group after transduction with each of the 4 HOXA9shRNA constructs, revealing a differential effect on viability that is significantly more prominent in $M L L$-rearranged leukemia cells (Figure 4A). Next, we reasoned that induction of apoptosis via specific suppression of HOXA9 might manifest as a correlation between HOXA9 expression and cellular sensitivity. In an independent experiment, determination of baseline HOXA9 expression before HOXA9 suppression (Figure S9A) and analysis of viability after HOXA9shRNA transduction by MTT assay in a panel of AML and ALL cell lines (Figure S9B) identified a significant correlation between cell number and baseline HOXA9 mRNA expression, with the greatest effect on viability in cells expressing the highest HOXA9 levels $(\mathrm{R}=-0.8, P=.001$; Figure 4B). These data demonstrate that continued HOXA9 expression is important for leukemia cells expressing HOXA9 at high levels and further support the specificity of the shRNAmediated effects.

Next, the necessity of HOXA9 expression for survival of primary AML patient samples was assessed. First, we confirmed a high transduction efficiency $(\sim 80 \%)$ in primary AML cells by puromycin selection after transduction with control shRNA constructs (data not shown) and determined that HOXA9shRNA transduction efficiently suppressed HOXA9 expression compared with control (Figure S10). We then analyzed the effect of HOXA9 knockdown in $M L L$-rearranged and nonrearranged primary human AML cells (6 $M L L$-rearranged $6 M L L$ germline). Similar to our findings in cell lines and to previous publica- tions, ${ }^{14-16}$ we find significantly higher HOXA9 mRNA expression in $M L L$-rearranged AMLs ( $P=.004$; Figure 5A,B). Analysis of apoptosis induction by annexin $\mathrm{V}$ staining demonstrated a significant effect on $M L L$-rearranged leukemias with a mean viability/control of $91 \%$ in the $M L L$-germline group versus $31 \%$ in the $M L L$-rearranged group $(P=.010$; Figure $5 \mathrm{C}, \mathrm{D})$. Moreover, induction of apoptosis was significantly correlated with HOXA9 expression level before lentiviral transduction $(\mathrm{R}=-0.8, P=.005$; Figure $5 \mathrm{E})$. These data were also confirmed in independent repeat experiments and demonstrate that primary human $M L L$-rearranged AML samples remain dependent on HOXA9 expression for their survival.

\section{In vivo assessment of HOXA9 suppression}

Next, we assessed the in vivo effect of HOXA9 suppression by transplanting luciferase-positive $\mathrm{t}(4 ; 11)$ SEMK2 cells into SCIDbeige mice followed by subsequent in vivo bioluminescent imaging. In 2 independent experiments, 5 million SEMK2 cells/mouse were transduced with either the 1F3-HOXA9shRNA construct or GFP control shRNA and injected intravenously 24 hours after transduction. Injection of equal numbers of transplanted cells was confirmed by determination of total body luminescence 2 hours after transplantation, and there was no significant difference (Figure 6A). Longitudinal in vivo imaging revealed a significant difference in total body luminescence as early as 24 hours after transplantation $(P=.036)$. This difference increased progressively 
A

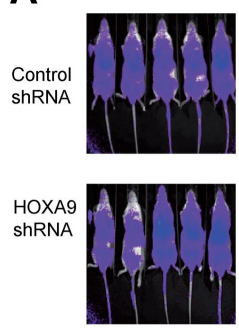

$2 \mathrm{~h}$

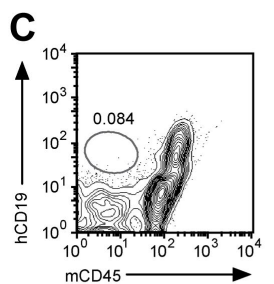

Wildtype SCID-beige

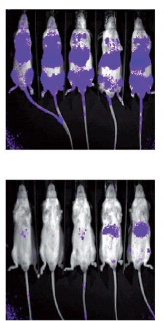

$72 \mathrm{~h}$

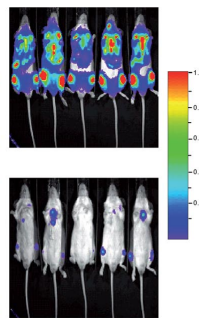

B

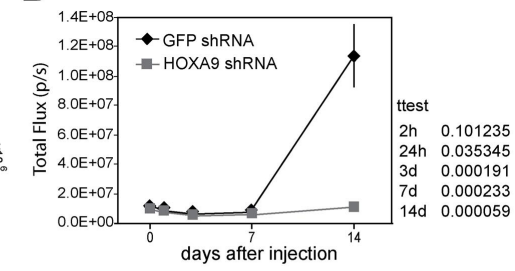

$14 d$
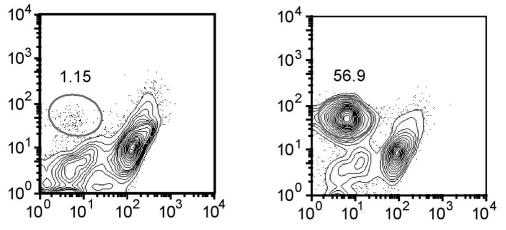

D

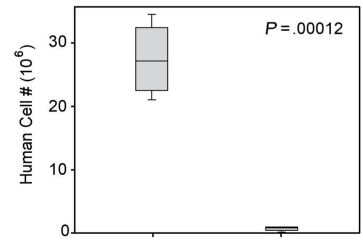

GFPshRNA 1F3-HOXA9 ShRNA
GFP shRNA

1F3-HOXA9 ShRNA

E

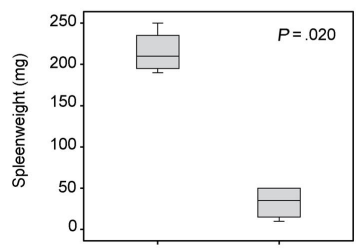

GFPshRNA 1F3-HOXA9 ShRNA

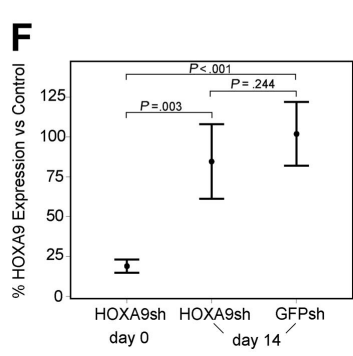

Figure 6. Assessment of the effects of HOXA9 suppression in vivo using bioluminescent imaging. (A) Before transplantation, SEMK2 cells were transduced with either 1F3-HOXA9 shRNA or GFP control shRNA and injected intravenously 24 hours after transduction. Mice were imaged 2,24 , and 72 hours after injection and then weekly. (B) There was no significant difference in total body luminescence 2 hours after injection confirming that an equal number of treated cells was injected $(P=.101)$. Repetitive longitudinal in vivo bioluminescent imaging at later time points revealed a significant difference of total body luminescence as early as 24 hours after transplantation $(P=.036)$. Fourteen days after transplantation, the differences in total body luminescence reached a significant maximum $(P=.001)$, shortly before the GFP shRNA-treated control group died of overt leukemia. (C) The degree of leukemic organ infiltration in both groups was assessed. The percentage of $h \mathrm{CD} 19^{+} / \mathrm{mCD} 5^{-}$SEMK2 cells was evaluated in spleens from wild-type SCID-beige mice that received 1F3-HOXA9-transduced or GFP-controltransduced cells. (D) The total number of human cells/spleen is graphed for both groups of mice. There is a 40 -fold difference in total number of SEMK2 between groups $(P=.001)$. (E) Spleen weights for are shown for mice that received either control or 1F3-HOXA9-transduced SEMK2 cells. (F) Analysis of HOXA9 expression in SEMK2 cells at the day of transplantation (day 0) confirmed efficient HOXA9 suppression (80\%) in the 1F3-HOXA9 shRNA-treated group compared with control shRNA-transduced cells. Human cells were isolated from mice that received either control or 1F3-HOXA9 shRNA-transduced SEMK2 cells 14 days after injection, and the HOXA9 expression level was compared with SEMK2 cells growing in culture. The HOXA9 expression level at this time point was similar in the sorted SEMK2 cells from the 1F3-HOXA9shRNA group as in those sorted from the GFP shRNA control group $(P=.244)$. over time with a maximum at day $14(P=.001)$ shortly before the mice in the GFP shRNA control group began to die of leukemia (Figure 6A,B). A subset of mice were killed at day 14 and assessed for the number of human cells present in spleens from each group of mice (Figure 6C). There was an approximately 40-fold difference in the number of human cells found in the spleens of the 2 groups of mice $(P=.001$; Figure 6D). Furthermore, there was a significant difference in the spleen weights between mice that received control-transduced cells and those that received HOXA9shRNA-transduced cells (Figure 6E). Thus, continued expression of HOXA9 is required for survival/proliferation of SEMK2 cells in vivo.

Even though there was a dramatic difference in the number of human cells present in the 2 groups of mice, some human cells could be found in the mice that received HOXA9shRNAtransduced SEMK2 cells (Figure 6C). We reasoned that the presence of these cells could indicate either the presence of a subpopulation of cells that do not require HOXA9 or the presence of cells that have escaped HOXA9 suppression. Thus, we sorted SEMK2 cells from both groups and assessed HOXA9 expression by quantitative real-time PCR. We compared expression levels to untreated SEMK2-M1 cells growing in culture and to SEMK2 cells 48 hours after transduction with the HOXA9shRNA construct. As expected, assessment before transplantation showed a significant decrease of HOXA9 expression in the transduced cells (Figure 6F). Interestingly, the cells sorted at day 14 from mice transplanted with HOXA9shRNA-transduced SEMK2 cells showed no significant difference compared with cells isolated from mice transplanted with GFP shRNA-transduced cells $(P=.244$; Figure $6 \mathrm{~F})$. This demonstrates that the few human cells found in the mice that received HOXA9shRNA-transduced SEMK2 cells have escaped HOXA9 suppression. Therefore, cells expressing high-level HOXA9 have a growth and survival advantage in vivo, and there does not appear to be a significant population of cells that are less dependent on HOXA9 expression for their survival/proliferation. These results were also confirmed applying a second HOXA9shRNA construct (2A5-HOXA9shRNA) in an independent in vivo experiment (Figure S11A-E). As in the in vivo experiment comparing 1F3HOXA9shRNA or GFP-control-shRNA transduced SEMK2 cells, human cells present in the spleens of SCID-beige mice transplanted with 2A5-HOXA9shRNA or GFP-control-shRNA-transduced SEMK2 cells were isolated at day 14. Assessment of HOXA9 expression showed no significant difference in both groups $(P=.195$; Figure S11D). Next, to determine whether the few isolated cells from the 2A5-HOXA9shRNA-transduced group were susceptible to subsequent HOXA9 suppression, cells were transduced with the 1F3-HOXA9shRNA construct or the GFPcontrol shRNA construct and viability in liquid culture was assessed. Seven days after transduction, a highly significant decreased number of viable cells was observed in cells transduced with the 1F3-HOXA9shRNA construct compared with cells transduced with the GFP-control shRNA $(P=.001$; Figure S11E). This demonstrates that the cells remained susceptible to HOXA9 suppression and further supports the notion that the small population of human cells found in the mice that received 2A5-HOXA9shRNAtransduced SEMK2 cells was not rendered HOXA9 independent but has rather escaped HOXA9 suppression in vivo.

\section{Discussion}

Comparative analysis of gene expression profiles in human acute myeloid and lymphoblastic leukemias implicates upregulation of a subset of $H O X$ genes as a central mechanism of 
leukemic transformation by MLL oncoproteins. ${ }^{14-16,21,22,61,62}$ Gene expression studies in murine models also demonstrate high-level expression of Hox genes in Mll-fusion initiated murine leukemias and leukemia stem cells. ${ }^{27,28}$ The central role for $H O X$ genes is further supported by recent studies that have identified $H O X$ genes as direct binding targets for wild-type MLL and MLL-fusion proteins. ${ }^{30-32}$ However, experiments in murine leukemia models directly assessing the requirement for specific Hox genes in Mll-rearranged leukemias have produced mixed results, perhaps because the Hox gene of interest is most often deleted in the germline and thus not present during leukemia initiation. Such models assess the necessity for a gene product to initiate leukemia, but they may not assess the continued requirement of a particular gene in a fully developed leukemia.

To further investigate the possibility that HOXA9 is necessary for survival of human leukemias, we used a lentiviral-based shRNA approach to inhibit HOXA9 in human leukemia cell lines and primary AML patient samples. We demonstrate that HOXA9 depletion leads to an almost immediate reduction of proliferative capacity, differentiation, and subsequent progressive induction of apoptosis, which was rescued by ectopic overexpression of HOXA9. The effect on apoptosis induction was significantly more pronounced in cells carrying an MLLfusion than in cells with MLL germline karyotype; however, even MLL germline cells possessing high-level HOXA9 expression demonstrated enhanced sensitivity to HOXA9 suppression. Furthermore, we demonstrate that HOXA9 suppression is also required for appropriate proliferation/survival in vivo. Global expression analysis after HOXA9 suppression revealed a tight correlation between HOXA9 suppression and subsequent downregulation of a gene set previously identified to be overexpressed in murine MLL-leukemia stem cells and human MLLAML including 5'-HOXA cluster genes, the HOXA9 cofactors MEIS1 and PBX3, and the transcription factor MEF2C. Although part of the observed expression changes might be the result of an already initiated apoptosis program rather than a direct effect of HOXA9 suppression, these findings further support HOXA9 as a central downstream target of select MLL-fusion proteins, which contributes to the induction and maintenance of an inappropriate gene expression program also found in primary human AML and in leukemogenic progenitor populations. ${ }^{28,29}$ The fact that some but not all genes aberrantly expressed in murine MLL leukemia stem cells and primary human MLL leukemias are also part of the HOXA9 suppression signature implies that MLL-fusion oncogene-mediated leukemic transformation is also mediated by other dysregulated pathways independent from HOXA9.

Our data showing that transplantation of HOXA9 depleted, human $\mathrm{t}(4 ; 11)$ SEMK2 cells into SCID-beige mice revealed a significant decrease in leukemia burden in vivo supports the concept that HOXA9 is crucial for survival of $M L L$-rearranged leukemias. It has been proposed previously that there may be functional redundancy within members of the clustered $H O X$ genes such that suppression of any one HOXA cluster gene may be compensated for by the high-level expression of other HOXA genes. In contrast, the data presented here reveal a significant effect of HOXA9 suppression, which could not be compensated by other mechanisms to maintain cell proliferation/survival. We note in our gene expression analysis that expression of other $5^{\prime}$-HOXA cluster genes shows a significant decrease after HOXA9 suppression. Extensive differences in sequence between HOXA9 and other Homeobox genes in the shRNA-targeted regions and similar expression changes with 2 different HOXA9shRNAs suggest that the shRNAs are not directly suppressing other HOX genes. Therefore, the observed decrease of gene expression of other $H O X$ genes appears to be a result of decreased HOXA9 expression, and sustained HOXA9 gene expression is necessary for growth/survival of human $M L L$ rearranged leukemias.

Demonstration that continued HOXA9 expression is required for leukemia survival has potential therapeutic implications. Although it is well known that $H O X$ gene expression is a frequent characteristic of both human acute leukemias and some solid tumors, most notably ovarian carcinoma, it has remained unclear whether such expression is required to maintain tumor survival. Therefore, the therapeutic potential of $\mathrm{HOX}$-associated gene expression programs remained unclear. The demonstration that suppression of $H O X$ gene expression leads to growth arrest in human $M L L$-rearranged leukemia cell lines and primary patient samples and subsequent down-regulation of other leukemia associated genes prompts further study of methods to inhibit HOX gene function and their downstream-associated programs. Having identified a gene signature after HOXA9 suppression that is associated with the abrogation of survival of $M L L$ rearranged human leukemias, it might be possible to use this "HOXA9-associated leukemia abrogation signature" for gene expression-based screening approaches of small molecule libraries as previously successfully demonstrated. ${ }^{63-65}$ Another approach would combine in vivo RNA interference conjugated to monoclonal antibodies to specifically target leukemia cells and suppress HOXA9-dependent pathways necessary for leukemia survival. It has been demonstrated in a recent proof-of-principle experiment that this approach might be feasible. ${ }^{66}$ Such novel therapeutic approaches might prove particularly useful in high risk $M L L$-rearranged leukemias. A similar approach can now be used to assess the importance of $H O X$ gene expression in other tumor types.

\section{Acknowledgments}

We thank Elise Porter, Sara Swalnick, and Megan Smith for administrative assistance, other members of the Armstrong laboratory for helpful discussion, and Dr William Hahn and other members of the RNAi consortium for providing the shRNA constructs.

This work was supported by the Leukemia \& Lymphoma Society, the Richard and Susan Smith Foundation, and the National Cancer Institute (NCI P01 CA66996).

\section{Authorship}

Contribution: J.F. performed and designed research and wrote the paper; A.V.K. performed and designed research; M.C.S., R.W., and T.N.D. performed research; M.v.d.H.-E. and C.M.Z. contributed vital reagents; and A.L.K. and S.A.A. designed research and wrote the paper.

Conflict-of-interest disclosure: The authors declare no competing financial interests.

Correspondence: Scott A. Armstrong, Children's Hospital, Boston, Karp Family Research Laboratories, 1 Blackfan Circle, Boston, MA 02215; e-mail: Scott.Armstrong@ childrens.harvard.edu. 
From www.bloodjournal.org at Erasmus MC Medical Library on June 16, 2009. For personal use only.

\section{References}

1. Chen CS, Hilden JM, Frestedt J, et al. The chromosome 4q21 gene (AF-4/FEL) is widely expressed in normal tissues and shows breakpoint diversity in $\mathrm{t}(4 ; 11)(\mathrm{q} 21 ; \mathrm{q} 23)$ acute leukemia. Blood. 1993;82:1080-1085.

2. Janssen JW, Ludwig WD, Borkhardt A, et al. Prepre-B acute lymphoblastic leukemia: high frequency of alternatively spliced ALL1-AF4 transcripts and absence of minimal residual disease during complete remission. Blood. 1994;84:38353842.

3. Kaneko Y, Shikano T, Maseki N, et al. Clinical characteristics of infant acute leukemia with or without 11q23 translocations. Leukemia. 1988;2: 672-676.

4. Borkhardt A, Wuchter C, Viehmann S, et al. Infant acute lymphoblastic leukemia: combined cytogenetic, immunophenotypical and molecular analysis of 77 cases. Leukemia. 2002;16:1685-1690.

5. Pui CH, Behm FG, Raimondi SC, et al. Secondary acute myeloid leukemia in children treated for acute lymphoid leukemia. N Engl J Med. 1989; 321:136-142.

6. Super HJ, McCabe NR, Thirman MJ, et al. Rearrangements of the MLL gene in therapy-related acute myeloid leukemia in patients previously treated with agents targeting DNA-topoisomerase II. Blood. 1993;82:3705-3711.

7. Ohshima A, Miura I, Chubachi A, et al. 11q23 aberration is an additional chromosomal change in de novo acute leukemia after treatment with etoposide and mitoxantrone. Am J Hematol. 1996; 53:264-266.

8. Ayton PM, Cleary ML. Molecular mechanisms of leukemogenesis mediated by MLL fusion proteins. Oncogene. 2001;20:5695-5707.

9. Chen CS, Sorensen PH, Domer PH, et al. Molecular rearrangements on chromosome 11q23 predominate in infant acute lymphoblastic leukemia and are associated with specific biologic variables and poor outcome. Blood. 1993;81:23862393.

10. Heerema NA, Arthur DC, Sather H, et al. Cytogenetic features of infants less than 12 months of age at diagnosis of acute lymphoblastic leukemia: impact of the 11q23 breakpoint on outcome: a report of the Childrens Cancer Group. Blood. 1994;83:2274-2284.

11. Rubnitz JE, Link MP, Shuster JJ, et al. Frequency and prognostic significance of $\mathrm{HRX}$ rearrangements in infant acute lymphoblastic leukemia: a Pediatric Oncology Group study. Blood. 1994;84: 570-573.

12. Pui CH, Gaynon PS, Boyett JM, et al. Outcome of treatment in childhood acute lymphoblastic leukaemia with rearrangements of the 11q23 chromosomal region. Lancet. 2002;359:19091915.

13. Pui $\mathrm{CH}$, Campana D. Age-related differences in leukemia biology and prognosis: the paradigm of MLL-AF4-positive acute lymphoblastic leukemia. Leukemia. 2007;21:593-594.

14. Armstrong SA, Staunton JE, Silverman LB, et al. MLL translocations specify a distinct gene expression profile that distinguishes a unique leukemia. Nat Genet. 2002;30:41-47.

15. Yeoh EJ, Ross ME, Shurtleff SA, et al. Classification, subtype discovery, and prediction of outcome in pediatric acute lymphoblastic leukemia by gene expression profiling. Cancer Cell. 2002 1:133-143.

16. Ferrando AA, Armstrong SA, Neuberg DS, et al. Gene expression signatures in MLL-rearranged T-lineage and B-precursor acute leukemias: dominance of HOX dysregulation. Blood. 2003; 102:262-268.

17. Krivtsov AV, Feng Z, Lemieux ME, et al. H3K79 methylation profiles define murine and human
MLL-AF4 leukemias. Cancer Cell. 2008;14:355368.

18. Krivtsov AV, Armstrong SA. MLL translocations, histone modifications and leukaemia stem-cell development. Nat Rev Cancer. 2007;7:823-833.

19. Cheung N, Chan LC, Thompson A, Cleary ML, So CW. Protein arginine-methyltransferase-dependent oncogenesis. Nat Cell Biol. 2007;9:1208-1215.

20. Krivtsov AV, Feng Z, Lemieux ME, et al. H3K79 methylation profiles define murine and human MLL-AF4 leukemias. Cancer Cell. 2008;14:355368.

21. Rozovskaia T, Feinstein E, Mor O, et al. Upregulation of Meis1 and HoxA9 in acute lymphocytic leukemias with the $t(4: 11)$ abnormality. Oncogene. 2001;20:874-878.

22. Drabkin HA, Parsy C, Ferguson K, et al. Quantitative HOX expression in chromosomally defined subsets of acute myelogenous leukemia. Leukemia. 2002;16:186-195.

23. Lavau C, Luo RT, Du C, Thirman MJ. Retrovirusmediated gene transfer of MLL-ELL transforms primary myeloid progenitors and causes acute myeloid leukemias in mice. Proc Natl Acad Sci U S A. 2000;97:10984-10989.

24. Kumar AR, Hudson WA, Chen W, Nishiuchi R, Yao Q, Kersey JH. Hoxa9 influences the phenotype but not the incidence of MII-AF9 fusion gene leukemia. Blood. 2004;103:1823-1828.

25. So CW, Karsunky H, Passegue E, Cozzio A, Weissman IL, Cleary ML. MLL-GAS7 transforms multipotent hematopoietic progenitors and induces mixed lineage leukemias in mice. Cancer Cell. 2003;3:161-171.

26. Zeisig BB, Milne T, Garcia-Cuellar MP, et al. Hoxa9 and Meis1 are key targets for MLL-ENLmediated cellular immortalization. Mol Cell Biol. 2004;24:617-628.

27. Ayton PM, Cleary ML. Transformation of myeloid progenitors by MLL oncoproteins is dependent on Hoxa7 and Hoxa9. Genes Dev. 2003;17:22982307.

28. Krivtsov AV, Twomey D, Feng Z, et al. Transformation from committed progenitor to leukaemia stem cell initiated by MLL-AF9. Nature. 2006;442 818-822.

29. Somervaille TC, Cleary ML. Identification and characterization of leukemia stem cells in murine MLL-AF9 acute myeloid leukemia. Cancer Cell. 2006;10:257-268.

30. Milne TA, Briggs SD, Brock HW, et al. MLL targets SET domain methyltransferase activity to Hox gene promoters. Mol Cell. 2002;10:11071117.

31. Nakamura T, Mori T, Tada S, et al. ALL-1 is a histone methyltransferase that assembles a supercomplex of proteins involved in transcriptional regulation. Mol Cell. 2002;10:1119-1128.

32. Milne TA, Martin ME, Brock HW, Slany RK, Hess JL. Leukemogenic MLL fusion proteins bind across a broad region of the Hox a9 locus, promoting transcription and multiple histone modifications. Cancer Res. 2005;65:1136711374.

33. Guenther MG, Jenner RG, Chevalier B, et al Global and Hox-specific roles for the MLL1 methyltransferase. Proc Natl Acad Sci U S A. 2005; 102:8603-8608.

34. Borrow J, Shearman AM, Stanton VP Jr, et al. The $t(7 ; 11)(\mathrm{p} 15 ; \mathrm{p} 15)$ translocation in acute myeloid leukaemia fuses the genes for nucleoporin NUP98 and class I homeoprotein HOXA9. Nat Genet. 1996;12:159-167.

35. Nakamura T, Largaespada DA, Lee MP, et al. Fusion of the nucleoporin gene NUP98 to HOXA9 by the chromosome translocation $\mathrm{t}(7 ; 11)(\mathrm{p} 15$; p15) in human myeloid leukaemia. Nat Genet. 1996;12:154-158.
36. Calvo KR, Sykes DB, Pasillas MP, Kamps MP Nup98-HoxA9 immortalizes myeloid progenitors, enforces expression of Hoxa9, Hoxa7 and Meis1, and alters cytokine-specific responses in a manner similar to that induced by retroviral coexpression of Hoxa9 and Meis1. Oncogene. 2002;21: 4247-4256.

37. Kroon E, Thorsteinsdottir U, Mayotte N Nakamura T, Sauvageau G. NUP98-HOXA9 expression in hemopoietic stem cells induces chronic and acute myeloid leukemias in mice. EMBO J. 2001;20:350-361.

38. Buske C, Feuring-Buske M, Abramovich C, et al. Deregulated expression of HOXB4 enhances the primitive growth activity of human hematopoietic cells. Blood. 2002;100:862-868.

39. Nakamura T, Largaespada DA, Shaughnessy JD Jr, Jenkins NA, Copeland NG. Cooperative activation of Hoxa and Pbx1-related genes in murine myeloid leukaemias. Nat Genet. 1996;12:149153.

40. Sauvageau G, Thorsteinsdottir U, Eaves CJ, et al. Overexpression of HOXB4 in hematopoietic cells causes the selective expansion of more primitive populations in vitro and in vivo. Genes Dev. 1995:9:1753-1765.

41. Thorsteinsdottir U, Mamo A, Kroon E, et al. Overexpression of the myeloid leukemia-associated Hoxa9 gene in bone marrow cells induces stem cell expansion. Blood. 2002;99:121-129.

42. Thorsteinsdottir U, Kroon E, Jerome L, Blasi F, Sauvageau G. Defining roles for HOX and MEIS1 genes in induction of acute myeloid leukemia. Mol Cell Biol. 2001;21:224-234.

43. Daga A, Podesta M, Capra MC, Piaggio G, Frassoni F, Corte $\mathrm{G}$. The retroviral transduction of HOXC4 into human CD34(+) cells induces an in vitro expansion of clonogenic and early progenitors. Exp Hematol. 2000;28:569-574.

44. Wermuth PJ, Buchberg AM. Meis1-mediated apoptosis is caspase dependent and can be suppressed by coexpression of HoxA9 in murine and human cell lines. Blood. 2005;105: 1222-1230.

45. Sauvageau G, Lansdorp PM, Eaves CJ, et al. Differential expression of homeobox genes in functionally distinct CD34+ subpopulations of human bone marrow cells. Proc Natl Acad Sci U S A. 1994;91:12223-12227.

46. Lawrence HJ, Sauvageau G, Ahmadi N, et al. Stage- and lineage-specific expression of the HOXA10 homeobox gene in normal and leukemic hematopoietic cells. Exp Hematol. 1995;23:11601166.

47. Pineault $\mathrm{N}$, Helgason CD, Lawrence HJ, Humphries RK. Differential expression of Hox Meis1, and $\mathrm{Pbx} 1$ genes in primitive cells throughout murine hematopoietic ontogeny. Exp Hematol. 2002;30:49-57.

48. Izon DJ, Rozenfeld S, Fong ST, Komuves L, Largman C, Lawrence HJ. Loss of function of the homeobox gene Hoxa-9 perturbs early T-cell development and induces apoptosis in primitive thymocytes. Blood. 1998;92:383-393.

49. Lawrence HJ, Helgason CD, Sauvageau G, et al. Mice bearing a targeted interruption of the homeobox gene HOXA9 have defects in myeloid, erythroid, and lymphoid hematopoiesis. Blood. 1997;89:1922-1930.

50. Lawrence HJ, Christensen J, Fong S, et al. Loss of expression of the Hoxa-9 homeobox gene impairs the proliferation and repopulating ability of hematopoietic stem cells. Blood. 2005;106:3988-3994.

51. So CW, Karsunky H, Wong P, Weissman IL, Cleary ML. Leukemic transformation of hematopoietic progenitors by MLL-GAS7 in the absence of Hoxa7 or Hoxa9. Blood. 2004;103: 3192-3199.

52. Levis M, Tse KF, Smith BD, Garrett E, Small D. A 
From www.bloodjournal.org at Erasmus MC Medical Library on June 16, 2009. For personal use only.

FLT3 tyrosine kinase inhibitor is selectively cytotoxic to acute myeloid leukemia blasts harboring FLT3 internal tandem duplication mutations. Blood. 2001;98:885-887.

53. Armstrong SA, Kung AL, Mabon ME, et al. Inhibition of FLT3 in MLL: validation of a therapeutic target identified by gene expression based classification. Cancer Cell. 2003;3:173-183.

54. Reich M, Liefeld T, Gould J, Lerner J, Tamayo P, Mesirov JP. GenePattern 2.0. Nat Genet. 2006; 38:500-501.

55. Subramanian A, Tamayo P, Mootha VK, et al. Gene set enrichment analysis: a knowledgebased approach for interpreting genome-wide expression profiles. Proc Natl Acad Sci U S A. 2005;102:15545-15550.

56. Moffat J, Grueneberg DA, Yang X, et al. A lentiviral RNAi library for human and mouse genes applied to an arrayed viral high-content screen. Cell. 2006;124:1283-1298.
57. Bridge AJ, Pebernard S, Ducraux A, Nicoulaz AL, Iggo R. Induction of an interferon response by RNAi vectors in mammalian cells. Nat Genet. 2003;34:263-264.

58. Cullen BR. Enhancing and confirming the specificity of RNAi experiments. Nat Methods. 2006;3: 677-681.

59. Wang J, Iwasaki H, Krivtsov A, et al. Conditional MLL-CBP targets GMP and models therapy-related myeloproliferative disease. EMBO J. 2005; 24:368-381.

60. Ross ME, Mahfouz R, Onciu M, et al. Gene expression profiling of pediatric acute myelogenous leukemia. Blood. 2004;104:3679-3687.

61. Golub TR, Slonim DK, Tamayo P, et al. Molecular classification of cancer: class discovery and class prediction by gene expression monitoring. Science. 1999;286:531-537.

62. Giampaolo A, Felli N, Diverio D, et al. Expression pattern of HOXB6 homeobox gene in myelomonocytic differentiation and acute myeloid leukemia. Leukemia. 2002;16:1293-1301.

63. Stegmaier K, Corsello SM, Ross KN, Wong JS, Deangelo DJ, Golub TR. Gefitinib induces myeloid differentiation of acute myeloid leukemia. Blood. 2005;106:2841-2848.

64. Stegmaier K, Ross KN, Colavito SA, O'Malley S, Stockwell BR, Golub TR. Gene expression-based high-throughput screening (GE-HTS) and application to leukemia differentiation. Nat Genet. 2004;36:257-263.

65. Lamb J, Crawford ED, Peck D, et al. The Connectivity Map: using gene-expression signatures to connect small molecules, genes, and disease. Science. 2006;313:1929-1935.

66. Song E, Zhu P, Lee SK, et al. Antibody mediated in vivo delivery of small interfering RNAs via cell-surface receptors. Nat Biotechnol. 2005;23:709-717. 